\title{
Androgen Receptor Antagonists and Anti-Prostate Cancer Activities of Some Newly Synthesized Substituted Fused Pyrazolo-, Triazolo- and Thiazolo-Pyrimidine Derivatives
}

\section{Saleh A. Bahashwan ${ }^{1}$, Ahmed A. Fayed ${ }^{2,3, *}$, Mohamed A. Ramadan ${ }^{4}$, Abd El-Galil E. Amr ${ }^{3,5}$} and Naif O. Al-Harbi ${ }^{6}$

1 Pharmacology and Toxicology Department, College of Pharmacy, Taibah University, Almadinah Almunawarah 22624, Saudi Arabia; E-Mail: drsalehamb@yahoo.com

2 Respiratory Therapy Department, College of Medical Rehabilitation Sciences, Taibah University, Almadina Almanoara 22624, Saudi Arabia

3 National Research Center, Cairo, Dokki 12622, Egypt; E-Mail: aeamr1963@yahoo.com

4 Microbiology and Immunology Departments, College of Medicine, Taibah University, Almadinah Almunawarah 22624, Saudi Arabia; E-Mail: mmostafa201120@yahoo.com.

5 Pharmaceutical Chemistry Department, Drug Exploration and Development Chair (DEDC), College of Pharmacy, King Saud University, Riyadh 11451, Saudi Arabia

6 Pharmacology and Toxicology Department, College of Pharmacy, King Saud University, Riyadh 11451, Saudi Arabia; E-Mail: ouf.nabil@yahoo.com.

* Author to whom correspondence should be addressed; E-Mail: dr_ahmedfayed14@yahoo.com; Tel.: +966-148-460-008 (ext. 8196); Fax: +966-148-485-853.

External Editor: William Chi-shing Cho

Received: 6 September 2014; in revised form: 17 November 2014 / Accepted: 18 November 2014 / Published: 24 November 2014

Abstract: A series of substituted pyrazole, triazole and thiazole derivatives (2-13) were synthesized from 1-(naphtho[1,2- $d$ thiazol-2-yl)hydrazine as starting material and evaluated as androgen receptor antagonists and anti-prostate cancer agents. The newly synthesized compounds showed potent androgen receptor antagonists and anti-prostate cancer activities with low toxicity (lethal dose $50\left(\mathrm{LD}_{50}\right)$ ) comparable to Bicalutamide as reference drug. The structures of newly synthesized compounds were confirmed by IR, ${ }^{1} \mathrm{H}-\mathrm{NMR},{ }^{13} \mathrm{C}-\mathrm{NMR}$, and MS spectral data and elemental analysis. The detailed synthesis, spectroscopic data, $\mathrm{LD}_{50}$ values and pharmacological activities of the synthesized compounds are reported. 
Keywords: naphthalinothiazolohydrazine; pyrazolopyrimidine; thiazolopyrimidine; anticancer activities

\section{Introduction}

Cancer poses a serious human health problem despite much progress in understanding its biology and pharmacology. Consequently, the design of new structures employed as antitumor agents is one of the most urgent research areas in contemporary medicinal chemistry. Some new heterocyclic compounds containing a thiazole ring were synthesized and used as antibacterial, antifungal [1,2] and anti-inflammatory agents (chemokine-receptor antagonists) [3]. These derivatives are also well known for their pronounced inhibition of the growth of cytomegalovirus (HCMV)-human cytomegalovirus [4], and are used as corticotrophin-releasing hormone (CRH-R1) receptor antagonists (display antidepressant activity) [5]. Thiazolopyrimidine derivatives were studied as potential drug candidates with anticancer activities [6,7]. In a previous work, we reported that certain of our newly substituted heterocyclic compounds exhibited antitumor activities [8-10]. Pyrimidine has gained considerable attention because of its diversity in biological activity and widespread applications in pharmaceuticals fields [11,12]. For examples, pyrimidine derivatives are used as Tie-2 kinase inhibitors [13,14], Human immunodeficiency virus type-1 (HIV-1) inhibitors [15,16], antimalarials [17,18], adenosine A1 receptor antagonists [19], anticancer agents [20], analgesics [21], cardiovascular [22] and anti-allergic agents [23,24]. The potent biological activities of various vitamins and drugs [25-28] are primarily contributed by the presence of heterocyclic rings in their molecular make-up. In view of these reports and in continuation of our previous works in heterocyclic chemistry, we report here newly synthesized substituted pyrazole derivatives. The androgen receptor antagonist, and anti-prostate cancer activities for the synthesized compounds was evaluated using Bicalutamide as a reference drug.

\section{Results and Discussion}

\subsection{Chemistry}

In the present study, a series of substituted pyrazole, triazole and thiazole derivatives (2-13) were synthesized from 1-(naphtho[1,2- $d$ ] thiazol-2-yl)hydrazine (1) as starting material. The starting material 1 was synthesized according to the reported literature [29] (Scheme 1).

Scheme 1. Synthetic route of 1-(naphtho[1,2-d] thiazol-2-yl)hydrazine 1.

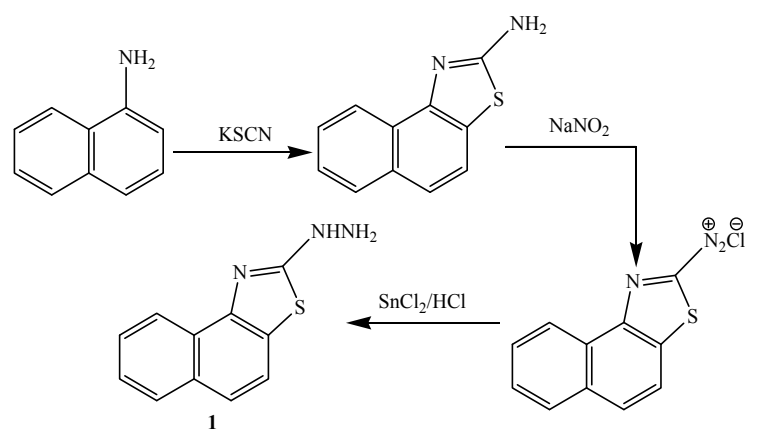


Treatment of $\mathbf{1}$ with ethoxymethylene malomonitrile afforded the corresponding 3-amino-4cyanopyrazolo derivatives $\mathbf{2}$, which was reacted with formamide or hydrazine hydrate to afford the corresponding 3-aminopyrazolopyrimidine 3 and aminopyrazolopyrazole derivative $\mathbf{4}$, respectively (Scheme 2).

Scheme 2. Synthetic route of compounds 2-4.

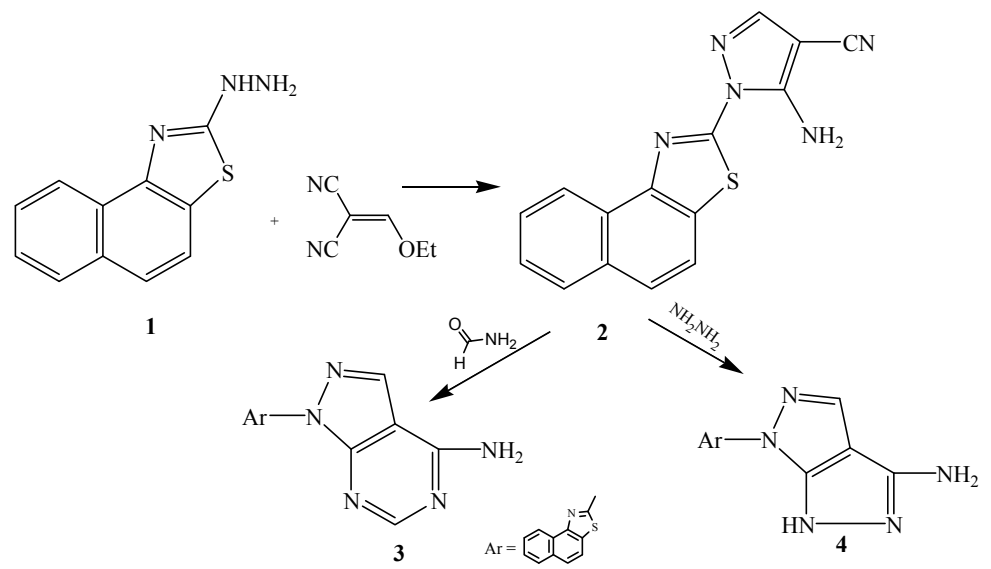

Compound 2 was reacted with ammonium isothiocyanate in the presence of ethanol to afford the corresponding pyrazolopyrimidine derivative 5. Also, compound $\mathbf{2}$ was treated with carbon disulphide or ethyl acetoacetate to afford the corresponding pyrazolothiazolino derivative 6 pyrazolopyridino derivative 7 , respectively (Scheme 3).

Scheme 3. Synthetic route of compounds 5-7.

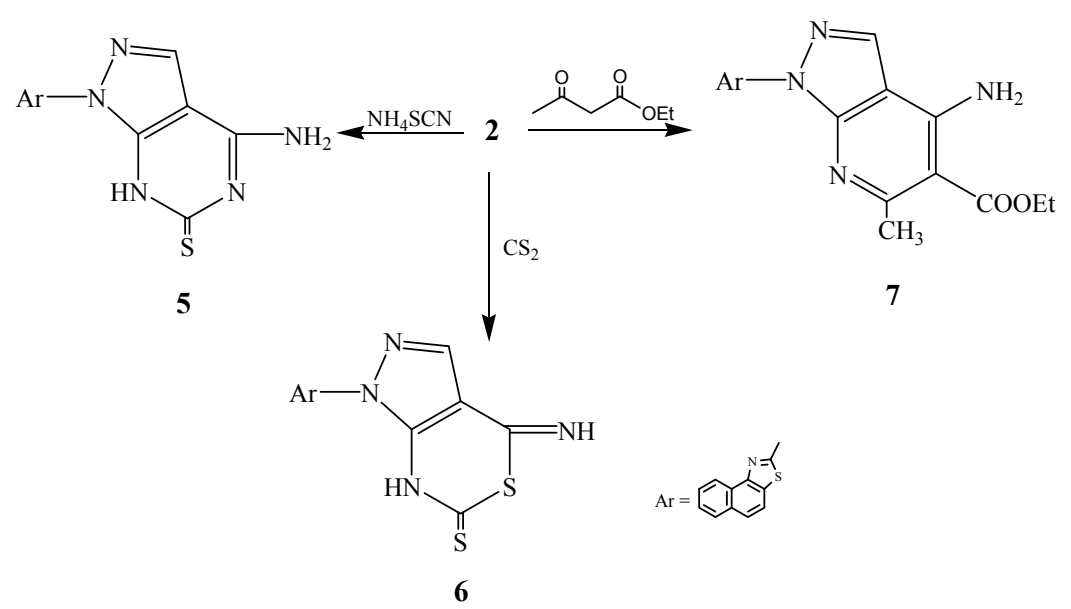

Additionally, compound $\mathbf{2}$ was reacted with triethylorthoformate in the presence of acetic anhydride to afford compound $\mathbf{8}$, which was reacted with hydrazine hydrate in the presence of acetic acid to afford the hydrazine derivative $\mathbf{9}$. The latter compound $\mathbf{9}$ was cyclized by acetic anhydride to the pyrazolotriazolopyrimidine derivative $\mathbf{1 0}$ (Scheme 4). 
Scheme 4. Synthetic route of compounds 8-10.

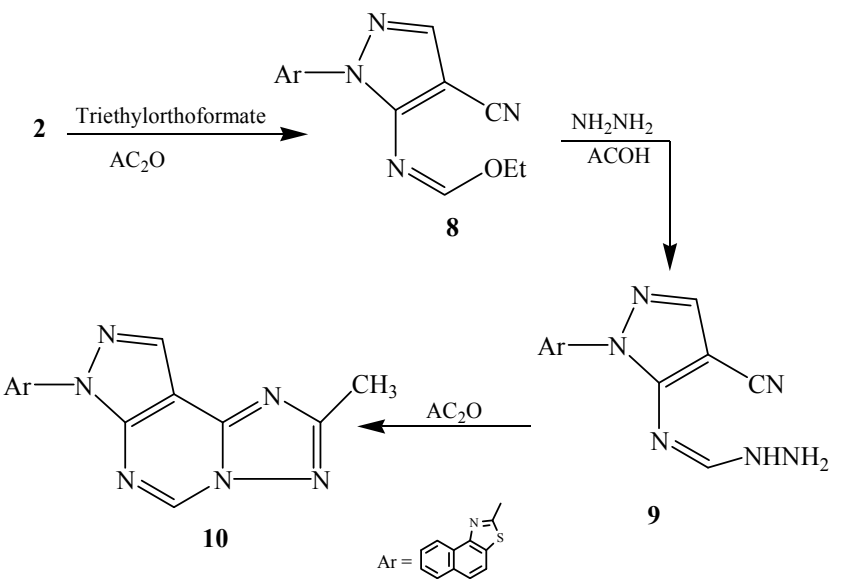

Finally, compound $\mathbf{8}$ was reacted with benzohydrazide or mercapto acetic acid to afford the corresponding compounds 11 and 12, respectively. Compound 12 was cyclized by refluxing with ethanolic sodium ethoxide to pyrazolothiazolopyrimidine derivative $\mathbf{1 3}$ (Scheme 5).

Scheme 5. Synthetic route of compounds 11-13.
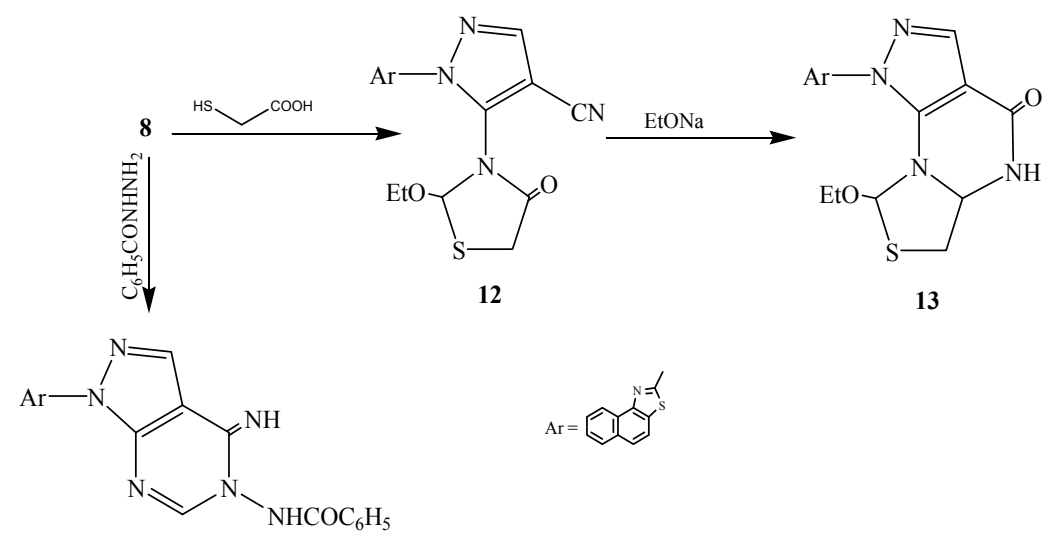

11

\subsection{Pharmacological Evaluation}

All the candidates were evaluated for their in vitro androgen receptor (AR) antagonistic activities using a reporter assay, and the resulting inhibitory concentration $\left(\mathrm{IC}_{50}\right)$ values are listed in Table 1. All new compounds were found to have potent in vitro AR antagonistic activities compared to Bicalutamide, but, compound $\mathbf{1}$ was devoid from any activities. Also, evaluated was the in vivo anti-androgenic activity in castrated immature rats, and the resulting percent inhibition values are listed in Table 2. The tested compounds were found to have potent in vivo anti-androgenic activities compared to Bicalutamide except compound $\mathbf{1}$ which was devoid from any activities.

In addition, all the analogues were evaluated by anti-prostate cancer screening anti-androgenic bioassay in human prostate cancer cells; the resulting $\mathrm{IC}_{50}$ values are listed in Table 3 . All tested newly synthesized compounds were found to have potent anti-prostate cancer activities compared to Bicalutamide except compound 1 which was devoid from any activities. The target compounds were tested for cytotoxicity against two human prostate cancer cell lines, lymph node carcinoma 
of the prostate (LNCaP) and human prostate cancer cell lines (PC-3). The LNCaP cell line is an androgen-dependent human prostate cancer cell line that expresses mutant AR, and the PC-3 cell line is an androgen-independent human prostate cancer cell line that does not express functional AR. All the tested compounds exhibited significant cytotoxicity in either LNCaP or PC-3 cells.

Table 1. In vitro androgen receptor antagonistic activities of the newly synthesized compounds.

\begin{tabular}{cc}
\hline Compound No. & IC $_{\mathbf{5 0}}(\boldsymbol{\mu M})$ \\
\hline Bicalutamide & 0.89 \\
$\mathbf{1}$ & Inactive \\
$\mathbf{2}$ & 0.031 \\
$\mathbf{3}$ & 0.037 \\
$\mathbf{4}$ & 0.034 \\
$\mathbf{5}$ & 0.041 \\
$\mathbf{6}$ & 0.044 \\
$\mathbf{7}$ & 0.057 \\
$\mathbf{8}$ & 0.077 \\
$\mathbf{9}$ & 0.075 \\
$\mathbf{1 0}$ & 0.023 \\
$\mathbf{1 1}$ & 0.039 \\
$\mathbf{1 2}$ & 0.091 \\
$\mathbf{1 3}$ & 0.029 \\
\hline
\end{tabular}

Table 2. In vivo anti-androgen activities of the newly synthesized compounds 1-13.

\begin{tabular}{ccc}
\hline Compound No. & \% $($ Inhibition) & ED $_{\mathbf{5 0}}(\mathbf{m g} / \mathbf{k g})$ \\
\hline Bicalutamide & 75 & 1.6 \\
$\mathbf{1}$ & Inactive & Inactive \\
$\mathbf{2}$ & 95.49 & 0.049 \\
$\mathbf{3}$ & 91 & 0.063 \\
$\mathbf{4}$ & 93.98 & 0.051 \\
$\mathbf{5}$ & 89.09 & 0.089 \\
$\mathbf{6}$ & 88.76 & 0.092 \\
$\mathbf{7}$ & 87.56 & 0.094 \\
$\mathbf{8}$ & 85.43 & 0.097 \\
$\mathbf{9}$ & 86.56 & 0.095 \\
$\mathbf{1 0}$ & 98.87 & 0.071 \\
$\mathbf{1 1}$ & 90.01 & 0.045 \\
$\mathbf{1 2}$ & 84.65 & 0.098 \\
$\mathbf{1 3}$ & 97.56 & 0.047 \\
\hline
\end{tabular}

Table 3. Anti-prostate cancer activities of the newly synthesized compounds $\mathbf{1}-\mathbf{1 3}$.

\begin{tabular}{ccc}
\hline Compound No. & IC $_{\mathbf{5 0}}(\mathbf{n M})$ PC-3 & IC $_{\mathbf{5 0}}(\mathbf{n M})$ LNCaP \\
\hline Bicalutamide & 822 & 619.00 \\
$\mathbf{1}$ & Inactive & Inactive \\
$\mathbf{2}$ & 156.78 & 51.47 \\
$\mathbf{3}$ & 177.45 & 58.58 \\
\hline
\end{tabular}


Table 3. Cont.

\begin{tabular}{ccc}
\hline Compound No. & IC $_{\mathbf{5 0}}(\mathbf{n M}) \mathbf{P C}-\mathbf{3}$ & $\mathbf{I C}_{\mathbf{5 0}}(\mathbf{n M}) \mathbf{L N C a P}$ \\
\hline $\mathbf{4}$ & 178.6 & 56.59 \\
$\mathbf{5}$ & 190.1 & 64.28 \\
$\mathbf{6}$ & 201.23 & 72.39 \\
$\mathbf{7}$ & 256.78 & 74.56 \\
$\mathbf{8}$ & 278.67 & 78.54 \\
$\mathbf{9}$ & 268.77 & 76.65 \\
$\mathbf{1 0}$ & 109.29 & 43.54 \\
$\mathbf{1 1}$ & 180.01 & 61.58 \\
$\mathbf{1 2}$ & 288.78 & 79.98 \\
$\mathbf{1 3}$ & 145.67 & 49.65 \\
\hline
\end{tabular}

2.3. Determination of Acute Toxicity (Lethal Dose 50 (LD50))

The $\mathrm{LD}_{50}$ was determined by using rats injected with different increasing doses of the synthesized compounds. The dose that killed $50 \%$ of the animals was calculated according to Austen and Brocklehurst [30] (Table 4).

Table 4. Acute toxicity ( $\mathrm{LD}_{50}$ ) of the synthesized of the newly synthesized compounds $\mathbf{1}-\mathbf{1 3}$.

\begin{tabular}{cc}
\hline Compound No. & LD $_{\mathbf{5 0}}(\mathbf{m g} / \mathbf{k g})$ \\
\hline $\mathbf{1}$ & 1164.45 \\
$\mathbf{2}$ & 163.46 \\
$\mathbf{3}$ & 174.56 \\
$\mathbf{4}$ & 265.67 \\
$\mathbf{5}$ & 266.58 \\
$\mathbf{6}$ & 257.79 \\
$\mathbf{7}$ & 146.80 \\
$\mathbf{8}$ & 235.99 \\
$\mathbf{9}$ & 124.89 \\
$\mathbf{1 0}$ & 315.78 \\
$\mathbf{1 1}$ & 324.57 \\
$\mathbf{1 2}$ & 234.46 \\
$\mathbf{1 3}$ & 443.35 \\
\hline
\end{tabular}

\section{Experimental Section}

\subsection{General Experimental Procedures}

Melting points were determined on open glass capillaries using an Electrothermal IA 9000 SERIES digital melting point apparatus (Electrothermal, Essex, UK), and are uncorrected. Elemental analyses were performed with all final compounds on an Elementar, Vario EL, Microanalytical Unit, National Research Centre, Cairo Egypt, and the results were found within $c a .0 .4 \%$ of the theoretical values. Analytical data were obtained from the Microanalytical Unit, Cairo University, Egypt. The IR spectra (KBr) were recorded on a FT IR-8201 PC spectrophotometer (Shimadzu, Tokyo, Japan). The ${ }^{1} \mathrm{H}-$ 
and ${ }^{13} \mathrm{C}$-NMR spectra were measured with a JEOL FTGNMEX 270, $270 \mathrm{MHz}$ instrument (JEOL, Tokyo, Japan) in DMSO- $d 6$, and chemical shifts were recorded in $(\delta, \mathrm{ppm})$ relative to tetramethylsilane (TMS). The mass spectra were run at $70 \mathrm{eV}$ with a Finnigan SSQ 7000 spectrometer (Thermo Electron Corporation, Madison, WI, USA.) using EI, and the values of $m / z$ are indicated in Dalton. TLC (Silica gel, aluminum sheets 60F254, Merck, Darmstadt, Germany) followed the reactions.

\subsection{Synthetic Procedures}

\subsubsection{Synthesis of 2-[3-Amino-4-cyanopyrazol-2-yl]naphthalino[1,2-d]thiazole (2)}

A mixture of compound $1(0.01 \mathrm{~mol})$ and ethoxymethylene malononitrile $(0.01 \mathrm{~mol})$ in absolute ethanol $(30 \mathrm{~mL})$ was refluxed for $2 \mathrm{~h}$. The solvent was evaporated under reduced pressure; the obtained solid was crystallized from ethanol to give the title product $\mathbf{2}$ as yellow powder. Yield: $87 \%$; m.p.: $256-258{ }^{\circ} \mathrm{C}$; IR $\left(\mathrm{KBr}, \mathrm{cm}^{-1}\right)$ : 3345-3315 $\left(\mathrm{NH}_{2}\right)$ and $2215(\mathrm{CN}) \mathrm{cm}^{-1}$; ${ }^{1} \mathrm{H}-\mathrm{NMR}$ (DMSO-d6): $\delta=6.78-7.61\left(\mathrm{~m}, 7 \mathrm{H}, 6 \mathrm{Ar}-\mathrm{H}\right.$ and $\left.1 \mathrm{H}_{\text {Pyrazolo }}\right)$ and $10.43\left(\mathrm{~b}, 2 \mathrm{H}, \mathrm{NH}_{2}\right.$ exchangeable with $\left.\mathrm{D}_{2} \mathrm{O}\right)$ ppm; ${ }^{13}$ C-NMR (DMSO- $d_{6}$ ): $\delta=112.2,112.2,113.6,118.2,119.4,127.1,128.5,129.1,134.4,143.5$ (Ar-C), $121.4(\mathrm{CN}), 131.2$ (thiazole-C), 132.4, 136.2, 142.3 (pyrazole-C) ppm; MS m/z (\%): $291\left(\mathrm{M}^{+}, 23\right)$, 265 (33), 249 (46), 212 (25), 184 (100), 126 (29), 102 (21), 78 (17). Anal. Calcd. for $\mathrm{C}_{15} \mathrm{H}_{9} \mathrm{~N}_{5} \mathrm{~S}$ (291.37): C, 61.82; H, 3.11; N, 24.04; S, 11.02. Found: C, 61.54; H, 2.96; N, 23.81; S, 10.82.

\subsubsection{Synthesis of 2-[6-Aminopyrimidino[4,5-c]pyrazol-2-yl]naphthalino[1,2- $d]$ thiazole (3)}

A solution of $2(0.01 \mathrm{~mol})$ in formamide $(20 \mathrm{~mL})$ was refluxed for $2 \mathrm{~h}$. After cooling, the precipitated solid product was collected by filtration, dried and crystallized from methanol to give the title product 3 as reddish brown powder. Yield: 66\%; m.p.: 290-292 ${ }^{\circ} \mathrm{C}$; IR $\left(\mathrm{KBr}, \mathrm{cm}^{-1}\right)$ : $3325\left(\mathrm{NH}_{2}\right) \mathrm{cm}^{-1} ;{ }^{1} \mathrm{H}-\mathrm{NMR}$ (DMSO- $\left.d_{6}\right): \delta=6.64-7.52(\mathrm{~m}, 8 \mathrm{H}, 6 \mathrm{Ar}-\mathrm{H}, 1$ HPyrazolo and 1HPyrimidino) and 11.12 (bs, 2H, $\mathrm{NH}_{2}$ exchangeable with $\mathrm{D}_{2} \mathrm{O}$ ) ppm; ${ }^{13} \mathrm{C}-\mathrm{NMR}$ (DMSO-d6): $\delta=112.1,113.6,118.2$, 119.4, 127.1, 128.5, 129.1, 134.4, 143.5, 145.4 (Ar-C), 142.3, 145.4, 149.1 (pyrazole-C), 154.1 (thiazole-C), 151.4, 151.5 (pyrimidine-C) ppm; MS m/z (\%): $318\left(\mathrm{M}^{+}, 19\right), 302$ (25), 276 (41), 212 (32), 184(47), 126 (100), 102 (37), 78 (14). Anal. Calcd. for $\mathrm{C}_{16} \mathrm{H}_{10} \mathrm{~N}_{6} \mathrm{~S}$ (318.40): C, 60.35; H, 3.16; N, 26.41; S, 10.08. Found: C, 60.14; H, 2.91; N, 26.27; S, 9.86.

\subsubsection{Synthesis of 2-[5-Amino-3H-pyrazolo[3,4-c]pyrazol-2-yl]naphthalino[1,2- $d]$ thiazole (4)}

A mixture of $2(0.01 \mathrm{~mol})$ and hydrazine hydrate $(0.01 \mathrm{~mol})$ in acetic acid $(30 \mathrm{~mL})$ was refluxed for $3 \mathrm{~h}$. The reaction mixture was poured onto ice-cold water. The obtained precipitate solid was filtered off, dried and crystallized from ethanol to give title product $\mathbf{4}$ as a green powder. Yield 69\%; m.p.: $>300{ }^{\circ} \mathrm{C}$; IR ( $\left.\mathrm{KBr}, \mathrm{cm}^{-1}\right): 3372-3323\left(\mathrm{NH}, \mathrm{NH}_{2}\right) \mathrm{cm}^{-1} ;{ }^{1} \mathrm{H}-\mathrm{NMR}\left(\mathrm{DMSO}-d_{6}\right): \delta=6.15$ (s, 1H, NH exchangeable with $\left.\mathrm{D}_{2} \mathrm{O}\right), 7.21-7.58(\mathrm{~m}, 7 \mathrm{H}, 6 \mathrm{Ar}-\mathrm{H}$ and $1 \mathrm{H}$ pyrazolo $)$ and $11.42\left(\mathrm{bs}, 2 \mathrm{H}, \mathrm{NH}_{2}\right.$ exchangeable with $\mathrm{D}_{2} \mathrm{O}$ ) ppm; ${ }^{13} \mathrm{C}-\mathrm{NMR}$ (DMSO- $d_{6}$ ): $\delta=111.5,112.7,116.2,118.2,125.5,129.9$, 131.4, 133.6, 144.4, 146.2 (Ar-C), 143.2, 145.3, 148.3, 152.3 (pyrazole-C), 151.4 (thiazole-C) ppm; MS m/z (\%): 306 (M+1,31), 290 (18), 261 (37), 225 (29), 184 (100), 145 (42), 108 (15), 50 (21). Anal. Calcd. for $\mathrm{C}_{15} \mathrm{H}_{10} \mathrm{~N}_{6} \mathrm{~S}$ (306.39): C, 58.79; H, 3.28; N, 27.43; S, 10.47. Found: C, 58.59; H, 3.11; N, 27.22; S, 10.24. 
3.2.4. Synthesis of 2-[6-Amino-3H-4-thiopyrimidino[4,5-c]pyrazol-2-yl]naphthalino[1,2-d]thiazole (5)

A mixture of $2(0.01 \mathrm{~mol})$ and ammonium isothiocyanate $(0.01 \mathrm{~mol})$ in dry acetone $(30 \mathrm{~mL})$ was refluxed for $2 \mathrm{~h}$ with stirring. The reaction mixture was concentrated under reduced pressure, the obtained solid was collected by filtration, dried and crystallized from ethanol to give the title product 5 as yellow crystals. Yield: $63 \%$; m.p.: $243-145{ }^{\circ} \mathrm{C}$; IR $\left(\mathrm{KBr}, \mathrm{cm}^{-1}\right): 3375-3315\left(\mathrm{NH}, \mathrm{NH}_{2}\right)$ and $1090(\mathrm{C}=\mathrm{S}) \mathrm{cm}^{-1} ;{ }^{1} \mathrm{H}-\mathrm{NMR}$ (DMSO- $\left.d_{6}\right): \delta=6.93-7.28\left(\mathrm{~m}, 7 \mathrm{H}, 6 \mathrm{Ar}-\mathrm{H}\right.$ and $\left.1 \mathrm{H}_{\text {Pyrazolo }}\right), 8.42(\mathrm{~s}, 1 \mathrm{H}$, $\mathrm{NH}$ exchangeable with $\mathrm{D}_{2} \mathrm{O}$ ) and 11.16 (bs, $2 \mathrm{H}, \mathrm{NH}_{2}$ exchangeable with $\mathrm{D}_{2} \mathrm{O}$ ) ppm; ${ }^{13} \mathrm{C}-\mathrm{NMR}$ (DMSO-d (D) $\delta=112.2,114.3,117.2,120.4,127.6,130.2,134.8,137.4,143.2,147.2$ (Ar-C), 132.5 $(\mathrm{C}=\mathrm{S}), 145.3$ (pyrazole-C), 148.6, 149.2, 152.3 (Pyrimidine-C), 150.3 (thiazole-C) ppm; MS m/z (\%): 350 (M+, 26), 334 (53), 264 (26), 225 (19), 199(28), 173 (33), 150 (100), 125 (26), 101 (19) 75 (21). Anal. Calcd. for $\mathrm{C}_{16} \mathrm{H}_{10} \mathrm{~N}_{6} \mathrm{~S}_{2}$ (350.50): C, 54.82; H, 2.87; N, 23.98; S, 18.31. Found: C, 54.57; H, 2.65; N, 23.64; S, 18.02 .

\subsubsection{Synthesis of 2-[6-Imino-3H-4-thiothiazino[4,5-c]pyrazol-2-yl]naphthalino[1,2- $d]$ thiazole (6)}

To compound $2(0.01 \mathrm{~mol})$, carbon disulfide $(15 \mathrm{~mL})$ was added drop-wise at room temperature. The reaction mixture was refluxed for $2 \mathrm{~h}$; after cooling, the obtained solid was filtered off, and crystallized from methanol to give the title product 6 as yellow powder. Yield: $59 \%$; m.p.: 264-266 ${ }^{\circ} \mathrm{C}$; IR $\left(\mathrm{KBr}, \mathrm{cm}^{-1}\right)$ : 3345-3290 (2 NH) and $1110(\mathrm{C}=\mathrm{S}) \mathrm{cm}^{-1} ;{ }^{1} \mathrm{H}-\mathrm{NMR}$ (DMSO-d $): \delta=5.62(\mathrm{~s}, 1 \mathrm{H}, \mathrm{NH}$ exchangeable with $\left.\mathrm{D}_{2} \mathrm{O}\right), 7.11-7.59\left(\mathrm{~m}, 7 \mathrm{H}, 6 \mathrm{Ar}-\mathrm{H}\right.$ and $\left.1 \mathrm{H}_{\text {Pyrazolo }}\right)$ and $9.16(\mathrm{~s}, 1 \mathrm{H}, \mathrm{NH}$ exchangeable with $\mathrm{D}_{2} \mathrm{O}$ ) ppm; ${ }^{13} \mathrm{C}-\mathrm{NMR}\left(\mathrm{DMSO}-d_{6}\right): \delta=113.3,114.9,116.7,119.1,126.5,131.5,135.3,136.5$, 142.6, 145.6 (Ar-C), $131.2(\mathrm{C}=\mathrm{S}), 146.4$ (pyrazole-C), 145.3, 148.4, 150.1 (thiazine-C), 152.7 (thiazole-C) ppm; MS m/z (\%): $367\left(\mathrm{M}^{+}, 35\right), 340$ (27), 308 (51), 249 (33), 225 (21), 167 (100), 126 (46), 102 (18), 89 (26) 76 (20). Anal. Calcd. for $\mathrm{C}_{16} \mathrm{H}_{9} \mathrm{~N}_{5} \mathrm{~S}_{3}$ (367.58): C, 52.27; H, 2.46; N, 19.05; S, 26.19. Found: C, 52.08; H, 2.22; N, 18.83; S, 26.02.

3.2.6. Synthesis of 2-[6-Amino-5-ethoxycarbonyl-4-methylpyridino[2,3-c]pyrazol-2-yl]naphthalino$[1,2-d]$ thiazole $(7)$

A mixture of $2(0.01 \mathrm{~mol})$ and ethyl acetoacetate $(0.01 \mathrm{~mol})$ in acetic acid $(30 \mathrm{~mL})$ in the presence of few drops of triethylamine was refluxed for $3 \mathrm{~h}$. The reaction mixture was poured into water, the separated solid was collected by filtration, dried and crystallized to give ethanol to give the title product 7 as reddish brown powder. Yield: $78 \%$; m.p.: over $300^{\circ} \mathrm{C}$; IR $\left(\mathrm{KBr}, \mathrm{cm}^{-1}\right): 3345-3110\left(\mathrm{NH}_{2}\right)$ and $1730\left(\mathrm{C}=\mathrm{O}\right.$ ester) $\mathrm{cm}^{-1}$; ${ }^{1} \mathrm{H}-\mathrm{NMR}\left(\mathrm{DMSO}-d_{6}\right): \delta=1.92\left(\mathrm{~s}, 3 \mathrm{H}, \mathrm{CH}_{3}\right), 2.43(\mathrm{t}, 3 \mathrm{H}, J=6.05 \mathrm{~Hz}$, $\left.\mathrm{CH}_{3}\right), 3.26\left(\mathrm{q}, 2 \mathrm{H}, J=7.05 \mathrm{~Hz}, \mathrm{CH}_{2}\right), 6.84-7.25\left(\mathrm{~m}, 7 \mathrm{H}, 6 \mathrm{Ar}-\mathrm{H}\right.$ and $1 \mathrm{H}_{\text {Pyrazolo }}$ ) and 11.26 (bs, 2H, $\mathrm{NH}_{2}$ exchangeable with $\left.\mathrm{D}_{2} \mathrm{O}\right)$ ppm; ${ }^{13} \mathrm{C}-\mathrm{NMR}$ (DMSO-d6): $\delta=24.2\left(\mathrm{CH}_{3}\right), 31.4\left(\mathrm{CH}_{3}\right), 63.4\left(\mathrm{CH}_{2}\right), 112.1$, 113.3, 118.5, 119.6, 120.4, 128.4, 132.3, 134.2, 143.4, 145.3 (Ar-C), 142.3, 144.6, 147.3, 148.9, 151.6 (pyridine-C), 150.1 (pyrazole-C), 152.2 (thiazole-C), $171.3(\mathrm{C}=\mathrm{O}) \mathrm{ppm}$; MS m/z (\%): $403\left(\mathrm{M}^{+}, 19\right)$, 372 (28), 343 (36), 299 (41 ), 263 (35), 225 (26), 184(34), 146 (100), 108 (23), 50 (14). Anal. Calcd. for $\mathrm{C}_{21} \mathrm{H}_{17} \mathrm{~N}_{5} \mathrm{SO}_{2}$ (403.49): C, 62.51; H, 4.24; N, 17.36; S, 7.95. Found: C, 62.28; H, 4.03; N, 17.18; $\mathrm{S}, 7.76$. 


\subsubsection{Synthesis of 2-[3-Ethylimidoformat-4-cyanopyrazol-2-yl]naphthalino[1,2-d]thiazole (8)}

A mixture of $2(0.01 \mathrm{~mol})$ and triethylorthoformate $(2.50 \mathrm{~mL})$ in acetic anhydride $(25 \mathrm{~mL})$ was heated under reflux for $2 \mathrm{~h}$. The reaction mixture was poured onto ice water, the formed solid product was collected by filtration, washed with water, dried and crystallized from ethanol to give the title product 8 as yellow crystals. Yield: 71\%; m.p.: 225-227 ${ }^{\circ} \mathrm{C}$; IR $\left(\mathrm{KBr}, \mathrm{cm}^{-1}\right): 2218(\mathrm{CN}) \mathrm{cm}^{-1}$; ${ }^{1} \mathrm{H}-\mathrm{NMR}$ (DMSO-d6): $\delta=1.62$ (t, 3H, CH $\left.3, J=6.05 \mathrm{~Hz}\right), 3.45$ (q, 2H, CH $\left.2, J=7.05 \mathrm{~Hz}\right), 4.27(\mathrm{~s}, 1 \mathrm{H}$, $\mathrm{CH}=\mathrm{N})$, and 7.14-7.35 (m, 7H, 6Ar-H and 1Hpyrazolo) ppm; ${ }^{13} \mathrm{C}-\mathrm{NMR}\left(\mathrm{DMSO}-d_{6}\right): \delta=20.2\left(\mathrm{CH}_{3}\right)$, $54.4\left(\mathrm{CH}_{2}\right), 113.6,114.3,116.2,118.7,120.5,126.7,128.1,130.5,133.2,142.4(\mathrm{Ar}-\mathrm{C}), 121.3(\mathrm{C}=\mathrm{N})$, $123.6(\mathrm{CN}), 134.7$ (thiazole-C), 133.5, 137.6, 141.4 (pyrazole-C) ppm; MS m/z (\%): $347\left(\mathrm{M}^{+}, 25\right)$, 321 (36), 276 (19), 248 (42), 225 (23), 184 (38), 126 (46), 92 (100) 76 (15). Anal. Calcd. for $\mathrm{C}_{18} \mathrm{H}_{13} \mathrm{~N}_{5} \mathrm{SO}$ (347.43): C, 62.22; H, 3.76; N, 20.16; S, 9.23. Found: C, 62.03; H, 3.52; N, 19.95; $\mathrm{S}, 9.05$.

\subsubsection{Synthesis of 2-[3-Imidoformic hydrazido-4-cyanopyrazol-2-yl]naphthalino[1,2- $d$ ]thiazole (9)}

A mixture of compound $8(0.01 \mathrm{~mol})$, and hydrazine hydrate $(0.01 \mathrm{~mol})$ in acetic acid $(20 \mathrm{~mL})$ was refluxed $2 \mathrm{~h}$. The obtained solid product was filtered off, dried and crystallized from methanol to give the title product 9 as a yellow powder. Yield: $62 \%$; m.p.: $212-214{ }^{\circ} \mathrm{C}$; IR $\left(\mathrm{KBr}, \mathrm{cm}^{-1}\right)$ : 3420-3265 (NH, $\left.\mathrm{NH}_{2}\right)$ and $2215(\mathrm{CN}) \mathrm{cm}^{-1} ;{ }^{1} \mathrm{H}-\mathrm{NMR}\left(\mathrm{DMSO}-d_{6}\right): \delta=4.12(\mathrm{~s}, 1 \mathrm{H}, \mathrm{CH}=\mathrm{N}), 6.12$ (bs, $2 \mathrm{H}, \mathrm{NH}_{2}$ exchangeable with $\left.\mathrm{D}_{2} \mathrm{O}\right), 6.59-7.18(\mathrm{~m}, 7 \mathrm{H}, 6 \mathrm{Ar}-\mathrm{H}$ and $1 \mathrm{H}$ Pyrazolo $)$ and $9.85(\mathrm{~b}, 1 \mathrm{H}, \mathrm{NH}$ exchangeable with $\mathrm{D}_{2} \mathrm{O}$ ) ppm; ${ }^{13} \mathrm{C}-\mathrm{NMR}$ (DMSO- $d_{6}$ ): $\delta=111.2,113.4,116.9,119.2,120.6,125.8$, 127.5, 131.3, 133.3, $140.4(\mathrm{Ar}-\mathrm{C}), 119.6(\mathrm{C}=\mathrm{N}), 125.3(\mathrm{CN}), 136.2$ (thiazole-C), 135.5, 139.3, 142.6 (pyrazole-C) ppm; MS m/z (\%): $333\left(\mathrm{M}^{+}, 21\right), 307$ (34), 276 (29), 249 (37), 212 (22), 184 (100), 146 (45), 108 (19), 50 (26). Anal. Calcd. for $\mathrm{C}_{16} \mathrm{H}_{11} \mathrm{~N}_{7} \mathrm{~S}$ (333.41): C, 57.63; H, 3.32; N, 29.41; S, 9.62. Found: C, 57.48; H, 3.11; N, 29.16; S, 9.35.

3.2.9. Synthesis of 2-[6-Methyl[1,2,4]triazolo[2,3-c]pyrimidino[6,5-c]pyrazol-2-yl]naphthalino[1,2d] thiazole (10)

A suspension of compound $9(0.01 \mathrm{~mol})$ in a mixture of acetic acid/acetic anhydride $(20 \mathrm{~mL} / 5 \mathrm{~mL})$ was heated under reflux for $2 \mathrm{~h}$. After cooling, the reaction mixture poured into water, the precipitated product was filtered off, washed with water, dried and crystallized from ethanol to give the title product 10 as green powder. Yield: 68\%; m.p.: 196-198 ${ }^{\circ} \mathrm{C} ;{ }^{1} \mathrm{H}-\mathrm{NMR}$ (DMSO- $d_{6}$ ): $\delta=2.15(\mathrm{~s}, 3 \mathrm{H}$, $\mathrm{CH}_{3}$ ), 4.23 (s, 1H, CH=N Pyrazolo), 6.59-7.18 (m, 7H, 6Ar-H, 1H Pyrimidino) ppm; ${ }^{13} \mathrm{C}-\mathrm{NMR}$ (DMSO- $d_{6}$ ): $\delta=21.5\left(\mathrm{CH}_{3}\right), 112.9,113.2,116.2,120.3,122.2,127.6,130.7,133.2,138.2,140.3(\mathrm{Ar}-\mathrm{C}), 143.6$, 145.5, 146.3, 148.5 (pyrimidine-C), 150.5 (triazole-C), 151.9 (pyrazole-C), 153.2 (thiazole-C) ppm; MS m/z (\%): 357 (M+32), 342 (42), 316 (29), 276 (36), 249 (52), 225 (21), 184 (19), 158 (100), 108 (25), 85 (17). Anal. Calcd. for $\mathrm{C}_{18} \mathrm{H}_{11} \mathrm{~N}_{7} \mathrm{~S}$ (357.43): C, 60.48; H, 3.10; N, 27.43; S, 8.98. Found: C, 60.19; H, 2.87; N, 27.13; S, 8.70. 
3.2.10. Synthesis of 2-[7-Benzamido-6-iminopyrimidino[4,5-c]pyrazol-2-yl]naphthalino[1,2- $d]$ thiazole (11)

A mixture of $8(0.01 \mathrm{~mol})$ and benzohydrazide $(0.01 \mathrm{~mol})$ in ethanol $(30 \mathrm{~mL})$ was refluxed $1 \mathrm{~h}$. The formed solid was collected by filtration, dried and crystallized from dioxane to give the title product 11 as yellowish powder. Yield: 59\%; m.p.: $276{ }^{\circ} \mathrm{C}$; IR $\left(\mathrm{KBr}, \mathrm{cm}^{-1}\right): 3395-3240(2 \mathrm{NH}) \mathrm{cm}^{-1}$; ${ }^{1} \mathrm{H}-\mathrm{NMR}$ (DMSO- $\left.d_{6}\right): \delta=5.37\left(\mathrm{~s}, 1 \mathrm{H}, \mathrm{NH}\right.$ exchangeable with $\left.\mathrm{D}_{2} \mathrm{O}\right), 6.59-7.18(\mathrm{~m}, 13 \mathrm{H}, 11 \mathrm{Ar}-\mathrm{H}$, 1Hpyrazolo and 1Hpyrimidino) and $9.31\left(\mathrm{~b}, 1 \mathrm{H}, \mathrm{NH}\right.$ exchangeable with $\mathrm{D}_{2} \mathrm{O}$ ) ppm; ${ }^{13} \mathrm{C}-\mathrm{NMR}$ (DMSO- $d 6$ ): $\delta=112.8,114.6,119.3,120.7,121.3,123.2,124.8,128.2,129.5,131.4,132.2,135.3,137.7,138.2$, 140.6, 142.3 (Ar-C), 144.6, 146.3, 149.5, 150.2 (pyrimidine-C), 151.4 (pyrazole-C), 152.2 (thiazole-C), $171.2\left(\mathrm{C}=\mathrm{O}\right.$, amide) ppm; MS m/z (\%): $437\left(\mathrm{M}^{+}, 27\right), 422$ (19), 345 (26), 317 (31), 261(53), 225 (29), 184 (36), 118 (100), 126 (32), 92 (17) 76 (24). Anal. Calcd. for $\mathrm{C}_{23} \mathrm{H}_{15} \mathrm{~N}_{7} \mathrm{SO}$ (437.52): C, 63.13; H, 3.45; N, 22.41; S, 7.33. Found: C, 62.91; H, 3.18; N, 22.14; S, 7.15.

3.2.11. Synthesis of 2-[3-(2-Ethoxy-4-oxo-5-dihydro-1,3-thiazol-3-yl)-4-cyanopyrazol-2-yl]naphthalino[1,2-d] thiazole (12)

A mixture of compound $8(0.01 \mathrm{~mol})$ and mercaptoacetic acid $(0.01 \mathrm{~mol})$ in dry benzene $(30 \mathrm{~mL})$ was stirred under reflux for $3 \mathrm{~h}$. After cooling, the obtained solid product was collected by filtration, washed with n-hexane, dried and crystallized from toluene to give the title product $\mathbf{1 2}$ as a yellow powder. Yield: $67 \%$; m.p.: $193{ }^{\circ} \mathrm{C}$; IR $\left(\mathrm{KBr}, \mathrm{cm}^{-1}\right): 2223(\mathrm{CN})$ and $1680(\mathrm{C}=\mathrm{O}$ amid $) \mathrm{cm}^{-1}$; ${ }^{1} \mathrm{H}-\mathrm{NMR}$ (DMSO-d6): $\delta=1.14$ (t, $\left.3 \mathrm{H}, J=6.05 \mathrm{~Hz}, \mathrm{CH}_{3}\right), 3.27$ (s, 2H, $\mathrm{CH}_{2}$ ), 3.41 (q, 2H, $J=7.05 \mathrm{~Hz}, \mathrm{CH}_{2}$ ), and 7.15-7.62 (m, 7H, 6Ar-H and1HPyrazolo) ppm; ${ }^{13} \mathrm{C}-\mathrm{NMR}$ (DMSO- $\left.d_{6}\right): \delta=22.5\left(\mathrm{CH}_{3}\right), 57.3\left(\mathrm{CH}_{2}\right)$, 110.8, 113.5, 116.4, 119.4, 120.6, 122.5, 126.2, 130.2, 132.3, 137.5 (Ar-C), $121.2(\mathrm{CN}), 144.2,146.4$, 151.1 (pyrazole-C), 145.2, 148.1, 150.3 (2 thiazole-C), $170.4(\mathrm{C}=\mathrm{O}) \mathrm{ppm}$; MS m/z $(\%): 420\left(\mathrm{M}^{+}, 36\right)$, 394 (21), 366 (42), 253 (31), 212 (28), 184 (100), 146 (37), 108 (25), 50 (19). Anal. Calcd. for $\mathrm{C}_{20} \mathrm{H}_{14} \mathrm{~N}_{5} \mathrm{~S}_{2} \mathrm{O}_{2}$ (420.56): C, 57.11; H, 3.35; N, 16.65; S, 7.61. Found: C, 56.87; H, 3.12; N, 16.39; S, 15.26 .

3.2.12. Synthesis of 2-[3-Ethoxy-5-dihydro-6H-7-oxo-[1,3]thiazolo[3,4-b]pyrimidino[4,5-c]pyrazol-2yl]naphthalino[1,2-d] thiazole (13)

A suspension of compound $12(0.01 \mathrm{~mol})$ in sodium ethoxide $(1.0 \mathrm{~g}$ sodium in $30 \mathrm{~mL}$ ethanol $)$ was heated under reflux for $3 \mathrm{~h}$. Excess of solvent was removed under reduced pressure to dryness, the residue was triturated with hot water. The formed solid was filtered off, washed with water, dried and crystallized from ethanol to give the title product $\mathbf{1 3}$ as a yellow powder. Yield: $72 \%$; m.p.: $181{ }^{\circ} \mathrm{C}$; IR $\left(\mathrm{KBr}, \mathrm{cm}^{-1}\right)$ : 3362-3215 (NH) and $1692\left(\mathrm{C}=\mathrm{O}\right.$ amide); ${ }^{1} \mathrm{H}-\mathrm{NMR}$ (DMSO- $\left.d_{6}\right): \delta=1.14(\mathrm{t}, 3 \mathrm{H}$, $\left.J=6.05 \mathrm{~Hz}, \mathrm{CH}_{3}\right), 3.27\left(\mathrm{~s}, 2 \mathrm{H}, \mathrm{CH}_{2}\right), 3.41\left(\mathrm{q}, 2 \mathrm{H}, J=7.05 \mathrm{~Hz}, \mathrm{CH}_{2}\right)$, and 7.15-7.62 (m, 7H, 6Ar-H and $\left.1 \mathrm{H}_{\text {Pyrazolo }}\right)$ and $10.12\left(\mathrm{~b}, 1 \mathrm{H}, \mathrm{NH}\right.$ exchangeable with $\left.\mathrm{D}_{2} \mathrm{O}\right) \mathrm{ppm} ;{ }^{13} \mathrm{C}-\mathrm{NMR}\left(\mathrm{DMSO}-d_{6}\right): \delta=21.2$ $\left(\mathrm{CH}_{3}\right), 54.3\left(\mathrm{CH}_{2}\right), 112.2,114.3,117.4,118.3,120.1,124.3,127.6,130.8,133.3,139.3(\mathrm{Ar}-\mathrm{C}), 144.6$, 147.3, 151.6 (pyrimidine-C), 150.4 (pyrazole-C), 146.5, 148.9, 151.4 (2 thiazole-C), $169.1(\mathrm{C}=\mathrm{O}$, amide) ppm; MS m/z (\%): $423\left(\mathrm{M}^{+}, 28\right), 378$ (52), 303 (19), 260 (22), 223 (41), 184 (100), 146 (37), 108 (28), 85 (35), 50 (19). Anal. Calcd. for $\mathrm{C}_{20} \mathrm{H}_{17} \mathrm{~N}_{5} \mathrm{~S}_{2} \mathrm{O}_{2}$ (423.40): C, 56.87; H, 4.05; N, 16.54; S, 15.12. Found: C, 56.65; H, 4.87; N, 16.35; S, 14.92. 


\subsection{Pharmacological Evaluation}

\subsubsection{Animals}

Albino rats (95) weighing 20-100 g were used and obtained from the Animal House Colony, Research Institute of Ophthalmology, Giza, Egypt. All animals (Guidelines for responsible conduct of research revised: march 2011 office of research integrity 132 cathedral of learning 412-624-3007) were maintained according to standard international human care and divided into fourteen equal groups. The animals were acclimatized for a period of two weeks in our laboratory environment prior to the study. They were housed in polypropylene cages, maintained under standard laboratory conditions, and fed with standard diet and water.

\subsubsection{Evaluation of Transcriptional Activity for Human Androgen Receptor}

\section{Establishment of Chinese Hamster Cell Model}

Chinese Hamster Ovary (CHO) Cells Stably Transfected with Human Androgen Receptor Gene and Mouse Mammary Tumor Virus (MMTV)-Luciferase Reporter Gene or SV40-Luciferase Gene: CHO cells were maintained in alpha-modified Eagle's medium supplemented with $10 \%$ fetal bovine serum (FBS). The culture medium of neomycin-resistant clone cells was supplemented with $10 \%$ dextran-coated charcoal-stripped FBS (DCC-FBS) and $500 \mathrm{mg} / \mathrm{mL}$ of neomycin. The CHO cells were transfected at $40 \%-70 \%$ confluence in $10-\mathrm{cm}$ petri dishes with a total of $20 \mathrm{mg}$ DNA (plasmide mammalian neow luciferase, (pMAMneoLUC)); Mouse mammary tumor virus (MMTV)-luciferase reporter plasmid and pSG5-hAR; human androgen receptor expression plasmid, or Simian vacuolating virus 40 (SV40-LUC); SV40-luciferase reporter plasmid containing neomycin resistant gene) by calcium phosphate mediated transfection. The stable transfected cells were selected in the culture medium supplemented with neomycin. The selected clone was designated as AR/CHO\#3 (human AR gene and MMTV luciferase reporter gene integrated CHO cell) or SV/CHO\#10 (SV-40-luciferase reporter gene integrated $\mathrm{CHO}$ cell), respectively [31].

Activities of the Tested Compounds to Inhibit Androgen Receptor Mediated Transcription Induced by Dihydrotestosterone (DHT) (AR Antagonistic Activity)

The stable transfected $\mathrm{AR} / \mathrm{CHO} \# 3$ or $\mathrm{SV} / \mathrm{CHO} \# 10$ cells were plated onto 96-well luminoplates (Packard, Palo Alto, CA, USA) at a density of $2 \times 104$ cells/well, respectively.

Four to eight hours later, the medium was changed to a medium containing DMSO, $0.3 \mathrm{nM}$ of DHT, or $0.3 \mathrm{nM}$ of DHT, and the tested compound. At the end of incubation, the medium was removed and the cells were lysed with $20 \mathrm{~mL}$ of lysis buffer $(25 \mathrm{mM}$ Tris- $\mathrm{HCl}$ (pH 7.8), $2 \mathrm{mM}$ dithiothreitol, $2 \mathrm{mM} \mathrm{1,2-cyclo-hexanediamine-tetraacetic} \mathrm{acid,} \mathrm{10 \%} \mathrm{glycerol} \mathrm{and} \mathrm{1 \%} \mathrm{Triton} \mathrm{X-100).}$ Luciferase substrate $(20 \mathrm{mM}$ Tris- $\mathrm{HCl}$ (pH 7.8), $1.07 \mathrm{mM}$ magnesium carbonate basic $\left(\mathrm{MgCO}_{3}\right)_{4} \mathrm{Mg}(\mathrm{OH})_{2} \cdot 5 \mathrm{H}_{2} \mathrm{O}, 2.67 \mathrm{mM} \mathrm{MgSO} 4 \cdot 7 \mathrm{H}_{2} \mathrm{O}, 0.1 \mathrm{mM}$ ethylenediaminetetraacetic acid (EDTA), $33.3 \mathrm{mM}$ dithiothreitol, $0.27 \mathrm{mM} \mathrm{CoA}, 0.47 \mathrm{mM}$ luciferin, $0.53 \mathrm{mM}$ ATP) was added, and luciferase activity was measured with a ML3000 luminometer (Dynatech Laboratories, El Paso, TX, UAS). AR antagonistic activities were calculated by the formula below: 


$$
\text { AR antagonistic activity }(\%)=100(I-X) /(I-B)
$$

I: (luciferase activity of $\mathrm{AR} / \mathrm{CHO} 33) /($ luciferase activity of $\mathrm{SV} / \mathrm{CHO \# 10)}$ in the presence of $0.3 \mathrm{nM}$ of DHT, B: (luciferase activity of AR/CHO\#3)/(luciferase activity of SV/CHO\#10) in the presence of DMSO, $X$ : (luciferase activity of $\mathrm{AR} / \mathrm{CHO} \# 3$ )/(luciferase activity of $\mathrm{SV} / \mathrm{CHO} \# 10$ ) in the presence of $0.3 \mathrm{nM}$ of DHT and the tested compound.

The concentration of compounds showing 50\% AR antagonistic activity, or IC50 values, was obtained by nonlinear analysis using a statistical analysis system (SAS).

\subsubsection{In Vivo Evaluation of Anti-Androgenic Activities in Castrated Immature Rats}

Androgen-treated male Wistar rats were obtained from the Animal House Colony, Research Institute of Ophthalmology, Giza, Egypt. Prepubertal male rats aged 3 weeks were castrated using the scrotal route under ether anesthesia. Three days after the castration, testosterone propionate (TP, $0.5 \mathrm{mg} / \mathrm{kg}$, was administered subcutaneously (s.c.) once daily for 5 days, alone or in combination with the tested compound (10-30 mg/kg, per os (p.o.)). TP was dissolved in cotton seed oil containing $5 \%$ ethanol. The tested compound was suspended with $0.5 \%$ methylcellulose. The rats were sacrificed by excessive chloroform anesthesia $6 \mathrm{~h}$ after final dosing, and both their ventral prostates and seminal vesicles-coagulate glands were removed and weighed. The anti-androgenic activity was expressed as a percentage of inhibition of the TP effect (TP-treated rats were arbitrarily assigned a value of $0 \%$ and vehicle-treated rats a value of $100 \%$ ) [31].

\subsubsection{Anti-Prostate Cancer Screening Anti-Androgenic Bioassay in Human Prostate Cancer Cells}

Human prostate cancer LNCaP and PC-3 cells were maintained in RPMI medium and Dulbecco's minimum essential medium (DMEM), respectively. Both media were supplemented with penicillin (25 units $/ \mathrm{mL}$ ), streptomycin $(25 \mathrm{mg} / \mathrm{mL}$ ), and 10\% fetal calf serum. For the androgen receptor transactivation assay, an androgen-dependent reporter gene transcription test was employed as the primary screening for potential anti-androgen identification. This assay was first performed in LNCaP cells, which express a clinically relevant mutant AR. Once anti-androgenic activity was detected in the LNCaP AR transactivation assay, compounds were re-examined for their potential activity against wild type AR. Wild type AR transactivation assay was performed in PC-3 host cells, which lack an endogenous, functional AR. The method and conditions of cell and gene transfection have been described previously. In brief, cells were plated in 24-well tissue culture dishes for 24 (PC-3 cells) or 48 (LNCaP cells) h prior to transfection. Subsequently, LNCaP cells were transfected with a reporter gene, MMTV-luciferase, which contains a MMTV-long terminal repeat (LTR) promoter and an androgen receptor binding element, and Renilla Luciferase Control Reporter Vectors 40 (PRL-SV40), which served as an internal control for transfection efficiency. PC-3 cells were transfected with a wild type AR expression plasmid, plasmide gene-5 androgen recptor (pSG5AR), in addition to the above-mentioned MMTV-luciferase reporter gene and PRL-SV40 internal control. SuperFect (Qiagen, Chatsworth, CA, USA) was employed as the transfection reagent following the manufacturer's recommendations. At the end of a 5-h transfection, the medium was changed 
to DMEM or RPMI supplemented with $10 \%$ charcoal dextran-stripped, i.e., androgen-depleted, serum. After $24 \mathrm{~h}$, the cells were treated with $1 \mathrm{nM}$ of DHT and/or test compounds at the designated concentrations.

After $24 \mathrm{~h}$ the cells were harvested for luciferase activity assay using the Dual Luciferase Assay System (Promega, Madison, WI, USA.). The derived data was expressed as relative luciferase activity normalized to the internal luciferase control. Cells cultured in medium containing DHT (androgen), as a positive control, induced a marked increase in reporter gene expression. Test compounds capable of significantly suppressing this DHT induced reporter gene expression were identified as potential anti-androgens [32].

\section{Structure Activity Relationship (SAR)}

Polycyclic ring systems highly increase activity, as observed in the cases of compounds 10, 12 and $\mathbf{1 3}$ that contain a polyhetrocyclic-fused ring system that provided potent activities.

Although compound $\mathbf{1}$ is a polyheterocyclic-fused ring system, it is completely free from any activity; this can lead to the conclusion that the pyrazoline ring moiety is essential for the activities.

A non-fused ring system, is present in compounds $\mathbf{1 2}$ and $\mathbf{9}$, which are less active than their polyheterocyclic-fused ring system chemical alternate derivatives $\mathbf{1 0}$ and $\mathbf{1 3}$ respectively. Also the separated ring system of derivative $\mathbf{1 2}$ was the least active one, perhaps due to electronic clouds and charge separation.

The free hydrazyl moiety plays an important role in intensifying the activity probably due to their proton accepting and electron donating functions; compound $\mathbf{9}$ is more active than compound $\mathbf{8}$.

Compound 4 that contains a bi-functional fused pyrazoline ring system is more active than those containing a pyrazoline-fused system with 6 member heterocyclic ring systems (compounds 3, 5, 6, 7, and 11).

Regarding the fusion of 6 member heterocyclic ring systems (compounds $\mathbf{3}, \mathbf{5}, \mathbf{6}, \mathbf{7}$ and 11) the descending orders of potency were $\mathbf{3}, \mathbf{1 1}, \mathbf{5}, \mathbf{6}$ and 7; this led to the conclusion that the 6 member ring system containing two hetero atoms is more active than compounds containing one hetero-atom; compounds 3, 5, 6 and 11 are more active than compound 7. Those containing two heteroatom's with a nitrogen atom were more active than compounds containing sulfur one; compounds $\mathbf{3 , 5}$ and $\mathbf{1 1}$ more active than compound $\mathbf{6}$.

\section{Conclusions}

The starting material $\mathbf{1}$ was used to synthesize the 3-amino-4-cyanopyrazolo derivative $\mathbf{2}$ which was treated with formamide or hydrazine hydrate to afford the corresponding aminopyrazolopyrimidine $\mathbf{3}$ and aminopyrazolopyrazole derivative $\mathbf{4}$, respectively. Compound $\mathbf{2}$ was treated with ammonium isothiocyanate, carbon disulfide or ethyl acetoacetate to afford compounds 5-7, respectively. Treatment of $\mathbf{2}$ with triethylorthoformate afforded derivative $\mathbf{8}$, which was treated with hydrazine hydrate to give 9 followed by cyclized with acetic anhydride to 10. Finally, compound $\mathbf{8}$ was reacted with benzoic acid hydrazide or mercapto acetic acid to give compounds $\mathbf{1 1}$ and 12, respectively. Compound 12 was cyclized with sodium ethoxide to afford the pyrazolothiazolopyrimidine derivative $\mathbf{1 3}$. The synthesized compounds were evaluated for their androgen receptor antagonists and anti-prostate cancer activities compared to that of Bicalutamide as a positive control. The acute toxicity of the compounds was assayed via the determination of their $\mathrm{LD}_{50}$. Most of the newly synthesized 
compounds were found to have potent $\mathrm{AR}$ antagonistic activities in vitro as compared with the reference drug Bicalutamide, while, compound $\mathbf{1}$ is devoid of any activity.

\section{Acknowledgments}

This work was fully supported by the Scientific Research Deanship, Taibah University, Al-Madina AL-Munawara, Kingdom of Saudi Arabia, Grant \#3667/434. The authors are grateful to Taibah University President and Dean of Scientific Research for their support.

\section{Author Contributions}

The listed authors contributed to this work as described in the following: Saleh A. Bahashwan gave the concepts of this work; Ahmed A. Fayed carried out of the synthetic work, interpreted the results and prepared the manuscript; Mohamed A. Ramadan interpreted the results of biological study; Abd El-Galil E. Amr and Naif O. Al-Harbi cooperated in the preparation of the manuscript. All authors read and approved the final manuscript.

\section{Conflicts of Interest}

The authors declare no conflict of interest.

\section{References}

1. Akbari, J.B.; Mehta, K.B.; Pathak, S.J.; Joshi, H.S. Synthesis and antimicrobial activity of some new pyrazolo[3,4- $d$ ]pyrimidines and thiazolo[4,5-d]pyrimidines. Indian J. Chem. 2008, 47, 477-480

2. Habib, N.S.; Soliman, R.; El-Tombary, A.A.; El-Hawash, S.A.; Shaaban, O.G. Synthesis of thiazolo[4,5-d]pyrimidine derivatives as potential antimicrobial agents. Arch. Pharm. Res. 2007, 30, 1511-1520.

3. Walters, I.; Austin, C.; Austin, R.; Bonnert, R.; Cage, P.; Christie, M.; Ebden, M.; Gardiner, S.; Grahames, C.; Hill, S.; et al. Evaluation of a series of bicyclic CXCR2 antagonists. Bioorg. Med. Chem. Lett. 2008, 18, 798-803.

4. Revankar, G.R.; Ojwang, J.O.; Mustain, S.D.; Rando, R.F.; de Clercq, E.; Huffman, J.H.; Drach, J.C.; Sommadossi, J.P.; Lewis, A.F. Thiazolo[4,5- $d]$ pyrimidines. Part II. Synthesis and anti-human cytomegalovirus activity in vitro of certain acyclonucleosides and acyclonucleotides derived from the guanine analogue 5-aminothiazolo [4,5- $d$ ] pyrimidine-2,7(3H,6H)-dione. Antivir. Chem. Chemother. 1998, 9, 53-63.

5. Beck, J.P.; Curry, M.A.; Chorvat, R.J.; Fitzgerald, L.W.; Gilligan, P.J.; Zaczek, R.; Trainor, G.L. Thiazolo[4,5-d]pyrimidine thiones and -ones as corticotropin-releasing hormone (CRH-R1) receptor antagonists. Bioorg. Med. Chem. Lett. 1999, 9, 1185-1188.

6. Rida, S.M.; Habib, N.S.; Badawey, E.A.; Fahmy, H.T.; Ghozlan, H.A. Synthesis of novel thiazolo[4,5-d]pyrimidine derivatives for antimicrobial, anti-HIV and anticancer investigation. Pharmazie 1996, 51, 927-931.

7. Fahmy, H.T.; Rostom, S.A.; Bekhit, A.A. Synthesis and antitumor evaluation of new polysubstituted thiazole and derived thiazolo[4,5-d]pyrimidine systems. Arch. Pharm. 2002, 335, 213-222. 
8. Hammam, A.G.; Fahmy, A.F.M.; Amr, A.E.; Mohamed, A.M. Synthesis of novel tricyclicheterocyclic compounds as potential anticancer agents using chromanone and thiochromanoneas synthons. Indian J. Chem. 2003, 42, 1985-1993.

9. Amr, A.G.; Mohamed, A.M.; Mohamed, S.F.; Abdel-Hafez, N.A.; Hammam, A.G. Anticancer activities of some newly synthesized pyridine, pyrane, and pyrimidine derivatives. Bioorg. Med. Chem. 2006, 14, 5481-5488.

10. Abou-Ghalia, M.H.; Amr, A.E. Synthesis and investigation of a new cyclo-( $N^{\alpha}$-dipicolinoyl) pentapeptide of a breast and CNS cytotoxic activity and an ionophoric specifity. Amino Acids 2004, 26, 283-289.

11. Abd El-Salam, O.I.; Fahmy, A.F.M.; Mohamed, A.M.; Elnaggar, D.H.; Hammam, A.G. Synthesis, anticancer and anti-inflammatory activities of 3,4-dihydro-7-nitrobenzo[b]oxepin-5(2H)one and its related derivatives. World J. Chem. 2010, 5, 7-17.

12. Borgio, J.F.; Bency, B.J.; Thorat, P.K.; Lonkar, A.D. Gynandropsis pentaphylla DC extracts on the production of microbial proteins. Am. J. Drug Discov. Dev. 2011, 1, 129-136.

13. Matloobi, M.; Kappe, C.O. Microwave-assisted solution- and solid-phase synthesis of 2-amino-4-arylpyrimidine derivatives. J. Comb. Chem. 2007, 9, 275-284.

14. Chengguo, L.V.; Lei, M.; Yan, S. Study on the diurnal changes of net photosynthetic rate and the impact factors of Stevia rebaudiana bertoni in autumn. Am. J. Plant Physiol. 2009, 4, 18-23.

15. Gadhachanda, V.R.; Wu, B.; Wang, Z.; Kuhen, K.L.; Caldwell, J.; Zondler, H.; Walter, H.; Havenhand, M.; He, Y. 4-Aminopyrimidines as novel HIV-1 inhibitors. Bioorg. Med. Chem. Lett. 2007, 17, 260-265.

16. Naeem, M.; Masroor, M.; Khan, A.; Morris, J.B. Agrobotanical attributes, nitrogen-fixation, enzyme activities and nutraceuticals of hyacinth bean (Lablab purpureus L.): A bio-functional medicinal legume. Am. J. Plant Physiol. 2009, 4, 58-69.

17. Negaoui, H.; Hanane, K.; Omar, K.; Djamel, S. A model of intestinal anaphylaxis in whey sensitized Balb/c mice. Am. J. Immunol. 2009, 5, 56-60.

18. Khan, M.S.H.; Wagatsuma, T.; Akhter, A.; Tawaraya, K. Sterol biosynthesis inhibition by paclobutrazol induces greater aluminum (Al) sensitivity in Al-tolerant rice. Am. J. Plant Physiol. 2009, 4, 89-99.

19. Chang, L.C.; Spanjersberg, R.F.; von Frijtag Drabbe Künzel, J.K.; Mulder-Krieger, T.; van den Hout, G.; Beukers, M.W.; Brussee, J.; Ijzerman, A.P. 2,4,6-Trisubstituted pyrimidines as a new class of selective adenosine A1 receptor antagonists. J. Med. Chem. 2004, 47, 6529-6540.

20. Capdeville, R.; Buchdunger, E.; Zimmermann, J.; Matter, A. Glivec (STI571, imatinib), a rationally developed, targeted anticancer drug. Nat. Rev. Drug Discov. 2002, 1, 493-502.

21. Rezvani, S.; Shariati, S. Effect of trehalose Arabidopsis thaliana L. on Huntington's disease. Am. J. Plant Physiol. 2010, 5, 1-6.

22. Hasanuzzaman, M.; Hossain, M.A.; Fujita, M. Physiological and biochemical mechanisms of nitric oxide induced abiotic stress tolerance in plants. Am. J. Plant Physiol. 2010, 5, 295-324.

23. Ozeki, K.; Ichikawa, T.; Takehara, H.; Tanimura, K.; Sato, M.; Yaginuma, H. Studies on antiallergy agents. III. Synthesis of 2-anilino-1,6-dihydro-6-oxo-5-pyrimidinecarboxylic acids and related compounds. Chem. Pharm. Bull. 1989, 37, 1780-1787. 
24. Dahmardeh, M. Effect of plant density and nitrogen rate on PAR absorption and maize yield. Am. J. Plant. Physiol. 2011, 6, 44-49.

25. Robin, A.; Julienne, K.; Meslin, J.C.; Deniaud, D. Synthesis of pyridone and pyridine rings by [4 + 2] heterocyclocondensation. Tetrahedron Lett. 2004, 45, 9557-9559.

26. Li, A.H.; Moro, S.; Forsyth, N.; Melman, N.; Ji, X.D.; Jacobson, K.A. Synthesis, CoMFA analysis, and receptor docking of 3,5-diacyl-2,4-dialkylpyridine derivatives as selective A3 adenosine receptor antagonists. J. Med. Chem. 1999, 42, 706-721.

27. Vacher, B.; Bonnaud, B.; Funes, P.; Jubault, N.; Koek. W.; Assié, M.B.; Cosi, C.; Kleven, M. Novel derivatives of 2-pyridinemethylamine as selective, potent, and orally active agonists at 5-HT1A receptors. J. Med. Chem. 1999, 42, 1648-1660.

28. Nasratun, M.; Said, H.A.; Noraziah A.; Alla, A.N.A. Immobilization of lipase from Candida rugosa on chitosan beads for transesterification reaction. Am. J. Appl. Sci. 2009, 6, 1653-1657.

29. Fayed, A.A.; Ghanem, S. Anti-microbial evaluation of new thiazolo pyrimidine derivatives. Eur. Sci. J. 2013, 9, 1857-7881.

30. Austen, K.F.; Brocklehurst, W.E. Anaphylaxis in chopped guinea pig lung. I. Effect of peptidase substrates and inhibitors. J. Exp. Med. 1961, 113, 521-539.

31. Kinoyama, I.; Taniguchi, N.; Kawaminami, E.; Nozawa, E.; Koutoku, H.; Furutani, T.; Kudoh, M.; Okada, M. N-Arylpiperazine-1-carboxamide derivatives: A novel series of orally active nonsteroidal androgen receptor antagonists. Chem. Pharm. Bull. 2005, 53, 402-409.

32. Lin, L.; Shi, Q.; Nyarko, A.K.; Bastow, K.F.; Wu, C.C.; Su, C.Y.; Shih, C.C.; Lee, K.H. Antitumor agents. 250. Design and synthesis of new curcumin analogues as potential anti-prostate cancer agents. J. Med. Chem. 2006, 49, 3963-3972.

(C) 2014 by the authors; licensee MDPI, Basel, Switzerland. This article is an open access article distributed under the terms and conditions of the Creative Commons Attribution license (http://creativecommons.org/licenses/by/4.0/). 